\title{
Geometric graph theory
}

\section{János Pach}

Summary A geometric graph is a graph drawn in the plane such that its vertices are points in general position and its edges are straight-line segments. The study of geometric graphs is a fairly new discipline abounding in open problems, but it has already yielded some striking results that have proved to be instrumental for the solution of various problems in combinatorial and computational geometry. These include the $k$-set problem, proximity questions, bounding the number of incidences between points and lines, designing various efficient graph drawing algorithms, etc. This paper surveys some Turán-type and Ramsey-type extremal problems for geometric graphs, and discusses their generalizations and applications.

\section{Introduction, basic definitions}

Let $G$ be a finite graph with no loops or multiple edges, whose vertex set and edge set are denoted by $V(G)$ and $E(G)$, respectively. By a drawing of $G$, we mean a representation of $G$ in the plane such that each vertex is represented by a distinct point and each edge by a simple (non-self-intersecting) continuous arc connecting the corresponding two points. If it is clear that we are referring to a drawing, and not to the underlying "abstract" graph, these points and arcs will also be called vertices and edges, respectively.

Two edges (arcs) cross each other if they have an interior point in common. This point is called a crossing. A crossing $p$ is called proper if in a small neighbourhood of $p$ one edge passes from one side of the other edge to the other side. We assume throughout that in a drawing

1. no edge passes through any vertex other than its endpoints,

2. no two edges touch each other (i.e., if two edges cross, then they properly cross),

3. no three edges cross at the same point.

A drawing in which every edge is represented by a straight-line segment is called a straight-line drawing or a geometric graph. Two geometric graphs are isomorphic if and only if one of them can be carried into the other using a rigid motion of the plane. We always assume that the vertices of a geometric graph are in general position, i.e. no three of them are collinear. If the vertices of a geometric graph are in convex position, i.e. they form the vertex set of a convex polygon, then the graph is called a convex geometric graph.

We emphasize that two edges of a geometric graph $G$ may cross each other. The number of crossings in $G$ is denoted by $\mathrm{CR}(G)$. Two edges of $G$ are disjoint if they do not cross and do not even share an endpoint. 
A geometric graph $G$ is said to be complete if its edge set consists of all $\left(\begin{array}{c}|V(G)| \\ 2\end{array}\right)$ segments between its vertices. If $V(G)=V_{1} \cup V_{2}$, and $E(G)$ consists of all segments between $V_{1}$ and $V_{2}$, then $G$ is a complete bipartite geometric graph. A geometric graph $G$ is said to be a geometric tree if the underlying abstract graph is a tree. A geometric graph $G$ is a geometric subgraph of another geometric graph $H$ if $V(G) \subseteq V(H)$ and $E(G) \subseteq E(H)$.

The systematic study of geometric graphs was initiated by $\mathrm{S}$. Avital and H. Hanani [7], P. Erdős, Y. Kupitz [48], and M. Perles. They realized that many classical questions in extremal graph theory [10] have natural analogues for geometric graphs. Some of these questions turned out to be surprisingly difficult and required new techniques combining geometric and combinatorial tools. In this paper we survey some recent results of this type and some tantalizing open problems.

Section 2 focuses on crossing-free geometric graphs. Section 3 describes some conditions which guarantee that a geometric graph determines many crossings. Sections 4,5 and 6 concentrate on Turán-type and Ramsey-type problems for geometric graphs, respectively. In Section 7 we present a few applications of our results, while Section 8 is devoted to geometric hypergraphs.

As usual, for any positive integer-valued functions, $f(n)$ and $g(n)$, we use the notations $f(n)=o(g(n)), f(n)=O(g(n))$, and $f(n)=\Omega(g(n))$ to indicate that $\lim _{n \rightarrow \infty} f(n) / g(n)=0, \sup f(n) / g(n)<\infty$, and $\inf f(n) / g(n)>0$, respectively. We write $f(n)=\Theta(g(n))$ if $f(n)=O(g(n))$ and $f(n)=\Omega(g(n))$.

For many other problems and results in this area, see [56] and Chapter 14 of $[57]$.

\section{Crossing-free geometric graphs}

Let $G=(V(G), E(G))$ be a geometric graph which has no crossing. Clearly, the underlying abstract graph, $G_{0}$, whose vertex set is $V(G)$ and whose edge set consists of those pairs of vertices which are connected in $G$ by a segment, is planar. Of course, the reverse is not true: the fact that $G_{0}$ is planar does not imply that $G$ must be crossing-free. However, by Fáry's theorem, for every abstract planar graph $H$, there is a crossing-free geometric graph $G$ whose underlying graph $G_{0}$ is isomorphic to $H$. In simpler terms, we have the following

Theorem 2.1 [24], [78], [71]. Every planar graph admits a crossing-free straight-line drawing.

We can modify the problem by putting certain restrictions on the drawing. For instance, given an abstract planar graph $H$ with $n$ vertices and a set $P$ of $n$ points in general position in the plane, we can ask whether there exists a crossing-free geometric graph $G$ with $V(G)=P$, whose underlying graph $G_{0}$ is isomorphic to $H$. To see that the answer is not necessarily in the affirmative, 
choose $H$ to be a complete graph with 4 vertices, and let $P$ consist of 4 points in convex position.

An abstract graph $H$ is called outerplanar if it can be drawn in the plane without crossing so that all points representing the vertices of $H$ lie on the boundary of the outer face of the resulting subdivision of the plane. Clearly, every tree is outerplanar. It follows from Theorem 2.1 that a graph is outerplanar if and only if it is the underlying graph of a crossing-free convex geometric graph (a triangulated cycle). However, these graphs also satisfy a stronger condition.

Theorem 2.2 [30]. For any outerplanar graph $H$ with $n$ vertices and for any set $P$ of $n$ points in the plane in general position, there is a crossing-free geometric graph $G$ with $V(G)=P$, whose underlying graph is isomorphic to $H$.

In other words, every outerplanar graph $H$ admits a straight-line drawing without crossing such that its vertex set is mapped into an arbitrarily prespecified $|V(H)|$-element set. Often we do not have much freedom in how to select this mapping. But sometimes we do. Perles conjectured that if $H$ is a tree, then we have at least one degree of freedom. More precisely, the following assertion is true.

Theorem 2.3 [63], [37]. Let $T$ be a rooted tree, and let $P$ be a set of $|V(T)|$ points in the plane in general position with a specified element $p \in P$.

Then $T$ admits a crossing-free straight-line drawing such that every vertex of $T$ is represented by an element of $P$, and the root is represented by $p$.

It may be conjectured that for any forest $F$ consisting of $k$ rooted trees $T_{1}, T_{2}, \ldots, T_{k}$ with roots $r_{1}, r_{2}, \ldots, r_{k}$, and for any set $P=\left\{p_{1}, p_{2}, \ldots, p_{K}\right\}$ of $K=\sum_{i=1}^{k}\left|T_{k}\right|$ points in general position in the plane, $F$ admits a crossing-free straight-line drawing such that every vertex of $F$ is represented by a point of $P$, and $r_{i}$ is represented by $p_{i}(i=1,2, \ldots, k)$. A. Kaneko and M. Kano [39], $[40],[41]$ verified this conjecture in several special cases, including when

1. $k=2$;

2. every tree $T_{i}$ is a rooted star, i.e., all of its vertices are adjacent to the root $r_{i}$

3. all trees $T_{i}$ are of the same size.

However, Kaneko and Kano believe that the above conjecture, in general, is false.

In some special cases, we may impose further restrictions on the drawings. For instance, colour the vertices of the tree $T$ with red and blue such that no two vertices of the same colour are adjacent. Let $n_{1}$ and $n_{2}$ denote the number 
of red and blue vertices, respectively. Given an $n_{1}$-element set $P_{1}$ and an $n_{2^{-}}$ element set $P_{2}$ in general position in the plane, we wish to decide whether there is a crossing-free straight-line drawing of $T$, whose red and blue vertices are represented by the elements of $P_{1}$ and $P_{2}$, respectively. In the case when $P_{1}$ and $P_{2}$ are separated by a straight-line, $n_{1}=n_{2}$, and $T$ is a path, the answer is in the affirmative [35].

Abellanas, García, Hernández, Noy and Ramos studied the following relaxation of this question: what happens if, instead of embedding a fixed tree $T$, we want to find a drawing of at least one member of a given class of trees?

Problem 2.4 [1]. Let $P_{1} \cup P_{2}$ be a point set in general position in the plane, $\left|P_{1}\right| \geq\left|P_{2}\right|>0$. Is it true that there always exists a crossing-free geometric tree on the vertex set $P_{1} \cup P_{2}$, whose every edge runs between $P_{1}$ and $P_{2}$, and in which the degree of every vertex is at most an absolute constant times $\left|P_{1}\right| /\left|P_{2}\right|$ ?

Abellanas et al. showed that the answer is in the affirmative if

1. $P_{1} \cup P_{2}$ is in convex position, or

2. $P_{1}$ and $P_{2}$ are separated by a straight line.

We close this section with a related question.

Problem 2.5 [25]. What is the size of the smallest point set $P$ in the plane with the property that for every abstract planar graph $H$ of $n$ vertices, there is a crossing-free geometric graph $G$, whose vertex set is a subset of $P$ and whose underlying graph is isomorphic to $H$ ?

The next theorem shows that this minimum is $O\left(n^{2}\right)$ (see also [68]).

Theorem 2.6 [25]. For every planar graph $H$, there is a crossing-free geometric graph whose vertex set is a subset of an $n$ by $2 n$ piece of the integer grid, and whose underlying graph is isomorphic to $H$.

\section{Unavoidable crossings}

The number of crossings in a geometric graph $G$ is denoted by $\mathrm{CR}(G)$. It follows from Euler's Polyhedral Formula that a geometric graph with $n$ vertices and more than $3 n-6$ edges cannot be crossing-free $(n \geq 3)$. In fact, this easily implies the following quantitative statement.

Lemma 3.1 Any geometric graph $G$ with $n \geq 3$ vertices and e edges has at least $e-3 n+6$ crossings. 
Proof By induction on $e$. If $e \leq 3 n-6$ then the statement is void. Assume $e>$ $3 n-6$. Then $G$ has at least one crossing. Applying the induction hypothesis to the graph $G^{\prime}$ obtained from $G$ by deleting an edge which participates in at least one crossing, we obtain that

$$
\left.\mathrm{CR}(G)=1+\mathrm{CR}\left(G^{\prime}\right) \leq 1+[(e-1)-3 n+6)\right]=e-3 n+6
$$

It is easy to see that Lemma 3.1 cannot be improved if $3 n-6 \leq e \leq 4 n-8$. However, as was shown by R. Eggleton and R. Guy [20], if $e>4 n-8$ then we can also find an edge which participates in at least two crossings. Using this fact, the above inductional argument yields that $G$ has at least

$$
[e-(3 n-6)]+[e-(4 n-8)]=2 e-7 n+14
$$

crossings.

This argument can be pushed a little further. Pach and G. Tóth proved the following.

Theorem 3.2 [65]. Let $G$ be a geometric graph with $n \geq 3$ vertices and $e$ edges such that every edge crosses at most $k$ others. Then, for $0 \leq k \leq 4$, we have

$$
e \leq(k+3)(n-2) \text {. }
$$

For $0 \leq k \leq 2$, this bound cannot be improved.

As before, this leads to a further improvement of Lemma 3.1.

Corollary 3.3 [65]. Any geometric graph $G$ with $n \geq 3$ vertices and e edges has at least

$$
\sum_{k=0}^{4}[e-(k+3)(n-2)]=5 e-25 n+50
$$

crossings. That is, we have $\mathrm{CR}(G) \geq 5 e-25+50$.

Ajtai, Chvátal, Newborn and Szemerédi [3] and independently T. Leighton [51] discovered that from Lemma 3.1 (or from the slightly stronger Corollary 3.3), by a simple trick, one can deduce a much better lower bound for the number of crossings in 'dense' graphs.

Theorem 3.4 [3], [51], [65]. Let $G$ be a geometric graph with $n$ vertices and $e$ edges. Then $\mathrm{CR}(G)$, the number of crossings in $G$, satisfies

$$
\mathrm{CR}(G) \geq \frac{1}{33.75} \frac{e^{3}}{n^{2}}-0.9 n>0.029 \frac{e^{3}}{n^{2}}-0.9 n .
$$


Proof Suppose first that $e \geq 7.5 n$. Construct a random geometric subgraph $G^{\prime} \subseteq G$ by selecting each vertex of $G$ independently with probability $p=$ $7.5 n / e \leq 1$, and letting $G^{\prime}$ be the geometric subgraph induced by the selected vertices. The expected number of vertices of $G^{\prime}, E\left[n^{\prime}\right]=p n$. Similarly, the expected number of edges and the expected number of crossings in $G^{\prime}$ satisfy $E\left[e^{\prime}\right]=p^{2} e$ and $E\left[\mathrm{CR}\left(G^{\prime}\right)\right]=p^{4} \mathrm{CR}(G)$, respectively.

Applying Corollary 3.3 to $G^{\prime}$ and taking expectations, we obtain

$$
p^{4} \mathrm{CR}(G)=E\left[\mathrm{CR}\left(G^{\prime}\right)\right]>5 E\left[e^{\prime}\right]-25 E\left[n^{\prime}\right]=5 p^{2} e-25 p n .
$$

This implies that

$$
\mathrm{CR}(G) \geq \frac{1}{33.75} \frac{e^{3}}{n^{2}}
$$

whenever $e \geq 7.5 n$. In fact, using Corollary 3.3 in the range $e<7.5 n$, it is easy to check that the slightly weaker inequality in Theorem 3.4 is valid for every geometric graph $G$.

Theorem 3.4 does not remain true if we replace the constant $1 / 33.75 \approx$ 0.029 by 0.06 . To see this, let $n \ll e \ll n^{2}$, and consider a geometric graph whose vertex set can be obtained by a slight perturbation of a $\lfloor\sqrt{n}\rfloor$ by $\lfloor\sqrt{n}\rfloor$ piece of the integer grid, and connect two gridpoints, $p$ and $q$, by an edge if and only if their distance satisfies

$$
|p-q| \leq \sqrt{\frac{2}{\pi} \cdot \frac{e}{n}}
$$

It is not hard to show that the number of crossings in this geometric graph is at most $0.06 e^{3} / n^{2}$.

One may believe that extending Theorem 3.2 to larger values of $k$ would result in a further improvement of the constant factor in Theorem 3.4. However, this is not the so. Although the estimate in Theorem 3.2 is tight for small values of $k$, it becomes weak as $k$ increases. It follows from Theorem 3.4 that if every edge of a geometric graph with $n$ vertices and $e$ edges participates in at most $k \geq 1$ crossings, then

$$
e \leq \sqrt{16.875 k} n \approx 4.108 \sqrt{k} n
$$

and this bound is tight apart from the value of the constant.

Although Theorem 3.4 is tight up to a constant factor, somewhat stronger estimates can be obtained for some special classes of geometric graphs. Let $\mathcal{K}_{r, s}$ denote the class of geometric graphs consisting of all $r s$ segments between some $r$-element set and some $s$-element set in the plane. In other words, $\mathcal{K}_{r, s}$ is the family of all complete bipartite geometric graphs with $r$ and $s$ vertices in their vertex classes. 
Theorem 3.5 [61]. Let $G$ be a geometric graph with $n$ vertices and $e \geq$ $4 n$ edges, which does not contain a complete bipartite geometric subgraph belonging to $\mathcal{K}_{r, s}$.

Then the number of crossings in $G$ satisfies

$$
\mathrm{CR}(G) \geq c_{r, s} \frac{e^{3+1 /(r-1)}}{n^{2+1 /(r-1)}},
$$

where $c_{r, s}>0$ is a suitable constant. These bounds are tight up to a constant factor, whenever $s>(r-1)$ !.

We close this section with another useful lower bound on the number of crossings in a geometric graphs. To state this result, we need a definition.

Definition Let $G_{0}$ be an abstract graph with vertex set $V\left(G_{0}\right)$ and edge set $E\left(G_{0}\right)$. The bisection width, $b\left(G_{0}\right)$, of $G_{0}$ is defined as the minimum number of edges, whose removal splits the graph into two roughly equal subgraphs. More precisely, $b\left(G_{0}\right)$ is the minimum number of edges running between $V_{1}$ and $V_{2}$, over all partitions of the vertex set of $G_{0}$ into two disjoint parts $V_{1} \cup V_{2}$ such that $\left|V_{1}\right|,\left|V_{2}\right| \geq\left|V\left(G_{0}\right)\right| / 3$.

The bisection width of a geometric graph $G$ is defined as the bisection width of the underlying abstract graph $G_{0}$, and is denoted by $b(G)$.

Leighton observed that there is an intimate relationship between the bisection width and the number of crossings in a geometric graph [51], which is based on the Lipton-Tarjan separator theorem for planar graphs [52]. The following version of this relationship was obtained by Pach, F. Shahrokhi, and M. Szegedy.

Theorem 3.6 [58]. Let $G$ be a geometric graph of $n$ vertices with degrees $d_{1}, d_{2}, \ldots, d_{n}$. Then

$$
\mathrm{CR}(G) \geq \frac{1}{40} b^{2}(G)-\frac{1}{16} \sum_{i=1}^{n} d_{i}^{2}
$$

\section{Forbidden geometric subgraphs - Multiple crossings}

In the spirit of Turán's classical theorem for abstract graphs [77], one can raise the following general question. Given a class $\mathcal{H}$ of so-called forbidden geometric subgraphs, what is the maximum number of edges that a geometric graph (resp. a convex geometric graph) of $n$ vertices can have without containing a geometric subgraph which belongs to $\mathcal{H}$ ? Denote this maximum by ex $(\mathcal{H}, n)$ (resp. by $\left.\operatorname{ex}_{c}(\mathcal{H}, n)\right)$.

For any $k \geq 2$, let $\mathcal{C}_{k}$ denote the class of all geometric graphs consisting of $k$ pairwise crossing edges. 
The simple fact that a crossing-free geometric graph of $n$ vertices has at most $3 n-6$ edges, can now be expressed by

$$
\operatorname{ex}\left(\mathcal{C}_{2}, n\right)=3 n-6
$$

for every $n \geq 3$. Similarly, inequality (1) can be rewritten as

$$
\operatorname{ex}\left(\mathcal{X}_{1, k}, n\right) \leq 4.108 \sqrt{k} n
$$

where $\mathcal{X}_{1, k}$ denotes the class of all geometric graphs consisting of $k+1$ edges such that one of them crosses the other $k$. (Note that $\mathcal{X}_{1,1}=\mathcal{C}_{2}$.)

The order of magnitude of the function ex $\left(\mathcal{C}_{3}, n\right)$ has been determined by Agarwal, Aronov, Pach, Pollack and Sharir.

Theorem 4.1 [2]. The maximum number of edges that a geometric graph of $n$ vertices can have without containing 3 pairwise crossing edges satisfies

$$
\operatorname{ex}\left(\mathcal{C}_{3}, n\right)=O(n)
$$

For $k \geq 4$, we do not know whether ex $\left(\mathcal{C}_{k}, n\right)$ is linear in $n$. However, as was shown by Pach, Shahrokhi and Szegedy, one can apply Theorems 3.6 and 4.1 to deduce that ex $\left(\mathcal{C}_{k}, n\right)$ is bounded from above by $n$ times a polylogarithmic factor.

Corollary 4.2 [58]. For every $k \geq 3$, we have

$$
\operatorname{ex}\left(\mathcal{C}_{k}, n\right)=O\left(n \log ^{2 k-6} n\right)
$$

Proof The assertion is true for $k=3$. We sketch the proof only for $k=4$. The other cases can be treated similarly.

Let $G$ be a geometric graph of $n$ vertices with degrees $d_{1}, d_{2}, \ldots, d_{n}$. Suppose that $G$ has no 4 pairwise crossing edges and $|E(G)|=\operatorname{ex}\left(\mathcal{C}_{4}, n\right)$. For every $e \in E(G)$, the edges crossed by $e$ form a geometric graph with no 3 pairwise crossing edges. Therefore, by Theorem 4.1, every edge crosses at most $O(n)$ other edges, and $\mathrm{CR}(G)=O(|E(G)| n)$. Since

$$
\sum_{i=1}^{n} d_{i}^{2} \leq\left(\sum_{i=1}^{n} d_{i}\right)\left(\max _{1 \leq i \leq n} d_{i}\right) \leq 2|E(G)| n
$$

it follows from Theorem 3.6 that $b(G)=O(\sqrt{|E(G)| n})$.

Consider a partition of $V(G)$ into two parts, $V_{1}$ and $V_{2}$, such that $\left|V_{1}\right|=$ $n_{1} \geq n / 3,\left|V_{2}\right|=n_{2} \geq n / 3$, and the number of edges connecting them is $b(G)$. Let $G_{1}$ and $G_{2}$ denote the geometric subgraphs of $G$ induced by $V_{1}$ and $V_{2}$, respectively. Since neither $G_{1}$ nor $G_{2}$ contains 4 pairwise crossing edges, we obtain

$$
|E(G)|=\left|E\left(G_{1}\right)\right|+\left|E\left(G_{2}\right)\right|+b(G) \leq \operatorname{ex}\left(\mathcal{C}_{4}, n_{1}\right)+\operatorname{ex}\left(\mathcal{C}_{4}, n_{2}\right)+O(|E(G)| n),
$$

which yields by an easy induction argument that

$$
|E(G)|=\operatorname{ex}\left(\mathcal{C}_{4}, n\right)=O\left(n \log ^{2} n\right) .
$$


Using the theory of generalized Davenport-Schinzel sequences [45], P. Valtr proved the following.

Theorem 4.3 [79]. Let $G$ be a geometric graph with $n$ vertices, all of whose edges can be intersected by a line. If $G$ has no $k$ pairwise crossing edges, then its number of edges is at most $c_{k} n$, where $c_{k}$ is a suitable constant depending only on $k$.

This result immediately implies that for every $k \geq 4$, the exponent $2 k-6$ of the logarithmic factor in Corollary 4.2 can be reduced to 1.

Corollary 4.4 [79]. For every $k \geq 4$, we have

$$
\operatorname{ex}\left(\mathcal{C}_{k}, n\right)=O(n \log n) .
$$

Proof Let $G$ be a geometric graph with $n$ vertices, with no $k$ pairwise crossing edges, and suppose that $|E(G)|=\operatorname{ex}\left(\mathcal{C}_{k}, n\right)$. Take a vertical line $l$ which does not pass through any of the vertices and which partitions the vertex set of $G$ into two roughly equal parts, $V_{1}$ and $V_{2}$, with $\left|V_{1}\right|=\lfloor n / 2\rfloor$ and $\left|V_{2}\right|=\lceil n / 2\rceil$. Let $G_{1}$ and $G_{2}$ denote the geometric subgraphs of $G$ induced by $G_{1}$ and $G_{2}$, respectively, and let $G^{\prime}$ be the geometric graph formed by the edges of $G$ intersecting $l$. Clearly, the number of edges of $G$ is equal to

$\operatorname{ex}\left(\mathcal{C}_{k}, n\right)=\left|E\left(G_{1}\right)\right|+\left|E\left(G_{2}\right)\right|+\left|E\left(G^{\prime}\right)\right| \leq \operatorname{ex}\left(\mathcal{C}_{k},\lfloor n / 2\rfloor\right)+\operatorname{ex}\left(\mathcal{C}_{k},\lceil n / 2\rceil\right)+c_{k} n$,

where we used Theorem 4.3 to estimate $\left|E\left(G^{\prime}\right)\right|$. The result follows.

It is conjectured that, for every fixed $k, \operatorname{ex}\left(\mathcal{C}_{k}, n\right)$ is linear in $n$. One can also make the following stronger conjecture.

Conjecture 4.5 For any $k \geq 3$, there exists an integer $C_{k}$ with the property that the edges of every geometric graph which has no $k$ pairwise crossing edges can be coloured by $C_{k}$ colours so that no two edges of the same colour cross each other.

A somewhat weaker conjecture which would also imply the linearity of $\operatorname{ex}\left(\mathcal{C}_{k}, n\right)$ is the following. For any $k \geq 3$, there exists a positive constant $\gamma_{k}$ such that every geometric graph without $k$ pairwise crossing edges has a crossing-free geometric subgraph which has at least $\gamma_{k}|E(G)|$ edges.

Unfortunately, we do not even know if Conjecture 4.5 is true for $k=3$. S. McGuinness [54] settled the case $k=3$ for every geometric graph whose edges can be intersected by a straight line. Conjecture 4.5 is also known to be true for convex geometric graphs. The following theorem of A. Kostochka and J. Kratochvíl slightly improves an old bound of A. Gyárfás [31],[32] (see also [33]) and Kostochka [46]. 
Theorem 4.6 [47]. Let $G$ be a convex geometric graph which has no $k$ pairwise crossing edges. Then one can colour the edges of $G$ with $2^{k+5}$ colours in such a way that no two edges of the same colour cross each other.

Kostochka [46] constructed a series of convex geometric graphs with no $k$ pairwise crossing edges, which cannot be coloured with fewer than a constant times $k \log k$ colours.

Theorem 4.6 plays an important role in the proof of Theorem 4.1. It also implies that $\operatorname{ex}_{c}\left(\mathcal{C}_{k}, n\right) \leq 2^{k+5}(2 n-3)=O(n)$ for every fixed $k$. However, $\mathrm{V}$. Capoyleas and Pach determined the exact value of $\operatorname{ex}_{c}\left(\mathcal{C}_{k}, n\right)$, which is one of the few non-trivial precise results in the area.

Theorem 4.7 [13]. Let $n \geq 2 k$. The maximum number of edges that a convex geometric graph of $n$ vertices can have without containing $k$ pairwise crossing edges satisfies

$$
\operatorname{ex}_{c}\left(\mathcal{C}_{k}, n\right)=2(k-1) n-\left(\begin{array}{c}
2 k-1 \\
2
\end{array}\right) .
$$

All of the results presented in this section can be rephrased in the following way. If a geometric graph $G$ with $n$ vertices has sufficiently many edges, then it must have a $k$-tuple of pairwise crossing edges. In fact, if $|E(G)|$ is very large, there are many such $k$-tuples. In the previous section we described this phenomenon for $k=2$ by a fairly tight quantitative formula. The proof of Theorem 3.4 may serve as a prototype of how to obtain many similar results.

Theorem 4.8 [55]. For any $k \geq 2$ there exists a constant $c(k)>0$ such that every convex geometric graph with $n$ vertices and $e \geq(2 k-1) n$ edges has at least $c(k) e^{2 k-1} / n^{2 k-2} \quad k$-tuples of pairwise crossing edges.

For $k=2$ and 3 , the last statement can be extended to all (not necessarily convex) geometric graphs. The same is true for every fixed $k$ such that ex $\left(\mathcal{C}_{k}, n\right)=O(n)$. By Theorem 4.8, if $G$ has constant times $n^{2}$ edges, then the total number of $k$-tuples of edges in $G$ is at least $c^{\prime} n^{2 k}$, for some $c^{\prime}>0$. A positive per cent of them are pairwise crossing.

\section{Forbidden geometric subgraphs - Non-crossing configurations}

In the previous section we discussed how many edges guarantee the existence of $k$ pairwise crossing edges in a geometric graph. Here we address the 'dual' question where, in place of pairwise crossing edges we look for pairwise disjoint edges. Recall that two disjoint edges cannot share even an endpoint.

For any $k \geq 2$, let $\mathcal{D}_{k}$ denote the class of all geometric graphs consisting of $k$ pairwise disjoint edges.

The following observation of Kupitz settles the question for convex geometric graphs. 
Theorem 5.1 [49]. Let $\operatorname{ex}_{c}\left(\mathcal{D}_{k}, n\right)$ denote the maximum number of edges that a convex geometric graph of $n$ vertices can have without containing $k$ pairwise disjoint edges. Then for every $k$ and $n \geq 2 k$, we have

$$
\operatorname{ex}_{c}\left(\mathcal{D}_{k}, n\right)=(k-1) n
$$

Proof Let $G$ be a convex geometric graph with $n$ vertices. Assume without loss of generality that $V(G)$ is the vertex set of a regular $n$-gon. Partition $E(G)$ into $n$ classes so that two segments belong to the same class if and only if they are parallel. If $G$ has no $k$ pairwise crossing edges then each class contains at most $k-1$ elements. Hence, $|E(G)| \leq(k-1) n$, and this bound can be attained.

The case of non-convex geometric graphs is more difficult. Hopf and Pannwitz [?] and (independently) Erdős proved a long time ago the following

Theorem 5.2 [36]. Let ex $\left(\mathcal{D}_{2}, n\right)$ denote the maximum number of edges that a geometric graph with $n$ vertices can have without containing two disjoint edges. Then, for every $n \geq 3$,

$$
\operatorname{ex}\left(\mathcal{D}_{2}, n\right)=n
$$

Proof (M. Perles) We say that an edge $x y$ is to the left of $x z$ if the ray $x z$ can be obtained from the ray $x y$ by a clockwise turn of less than $\pi$. A vertex $x$ is called pointed if all edges incident to it lie in a half-plane whose boundary contains $x$.

For every pointed vertex of $G$, delete the leftmost edge incident with it. If $G$ had no two disjoint edges then the resulting graph is empty. Indeed, if there were at least one remaining edge, $x z$, then we could find an edge $x y$ to the left of $x z$ and another edge $z v$ to the left of $z x=x z$, and these two edges would be disjoint. Since we deleted at most one edge for each vertex, $|E(G)| \leq|V(G)|$, and this bound can be attained.

Alon and Erdős [5] were the first to show the linearity of ex $\left(\mathcal{D}_{3}, n\right)$. The best known lower and upper bounds,

$$
2.5 n-4 \leq \operatorname{ex}\left(\mathcal{D}_{3}, n\right) \leq 3 n
$$

are due to Perles (unpublished) and Goddard, Katchalski and Kleitman [28], respectively. Recently, Tóth and Valtr [76] showed that

$$
4 n-9 \leq \operatorname{ex}\left(\mathcal{D}_{4}, n\right) \leq 8.5 n
$$

Pach and Törőcsik proved that ex $\left(\mathcal{D}_{k}, n\right)=O(n)$, for every fixed $k$. 


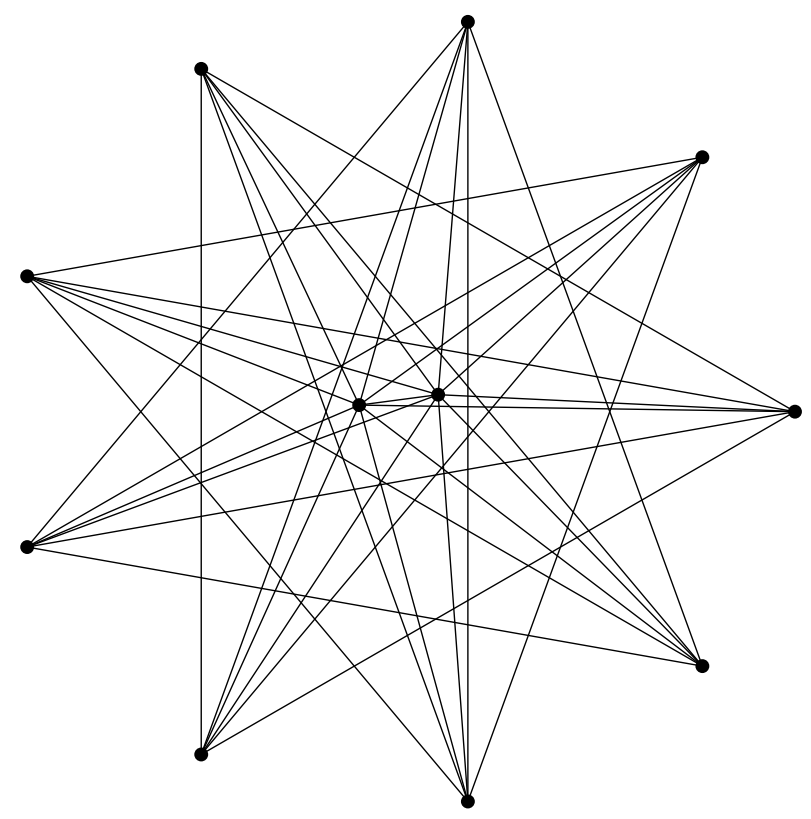

Figure 1: A geometric graph with no 4 pairwise disjoint edges.

Theorem 5.3 [64]. Let ex $\left(\mathcal{D}_{k}, n\right)$ denote the maximum number of edges that a geometric graph of $n$ vertices can have without containing $k$ pairwise disjoint edges. Then, for every $k$ and $n$, we have

$$
\operatorname{ex}\left(\mathcal{D}_{k}, n\right) \leq(k-1)^{4} n
$$

Proof Let $G$ be a geometric graph with $n$ vertices, containing no $k$ pairwise disjoint edges. For any vertex $v$, let $x(v)$ and $y(v)$ denote the $x$-coordinate and the $y$-coordinate of $v$, respectively. Assume without loss of generality that no two vertices have the same $x$-coordinate.

An edge $e \in E(G)$ is said to lie below another edge $e^{\prime} \in E(G)$ if there is no vertical line which intersects both $e$ and $e^{\prime}$, and whose intersection with $e^{\prime}$ is strictly below its intersection with $e$. (According to this definition, if the projections of $e$ and $e^{\prime}$ onto the $x$-axis do not overlap, then we say that $e$ lies below $e^{\prime}$ and also $e^{\prime}$ lies below $e$.)

Define four binary relations $\prec_{1}, \prec_{2}, \prec_{3}$, and $\prec_{4}$ on $E(G)$, as follows. One necessary condition for two edges to be in any of these relations is that they must be disjoint. Let $e=v_{1} v_{2}$ and $e^{\prime}=v_{1}^{\prime} v_{2}^{\prime}$ be two disjoint edges of $G$ with $x\left(v_{1}\right)<x\left(v_{2}\right)$ and $x\left(v_{1}^{\prime}\right)<x\left(v_{2}^{\prime}\right)$. Then

- $e \prec_{1} e^{\prime}$ if $x\left(v_{1}\right)<x\left(v_{1}^{\prime}\right), x\left(v_{2}\right)<x\left(v_{2}^{\prime}\right)$ and $e$ lies below $e^{\prime}$;

- $e \prec_{2} e^{\prime}$ if $x\left(v_{1}\right)>x\left(v_{1}^{\prime}\right), x\left(v_{2}\right)>x\left(v_{2}^{\prime}\right)$ and $e$ lies below $e^{\prime}$;

- $e \prec_{3} e^{\prime}$ if $x\left(v_{1}\right)<x\left(v_{1}^{\prime}\right), x\left(v_{2}\right)>x\left(v_{2}^{\prime}\right)$ and $e$ lies below $e^{\prime}$; 


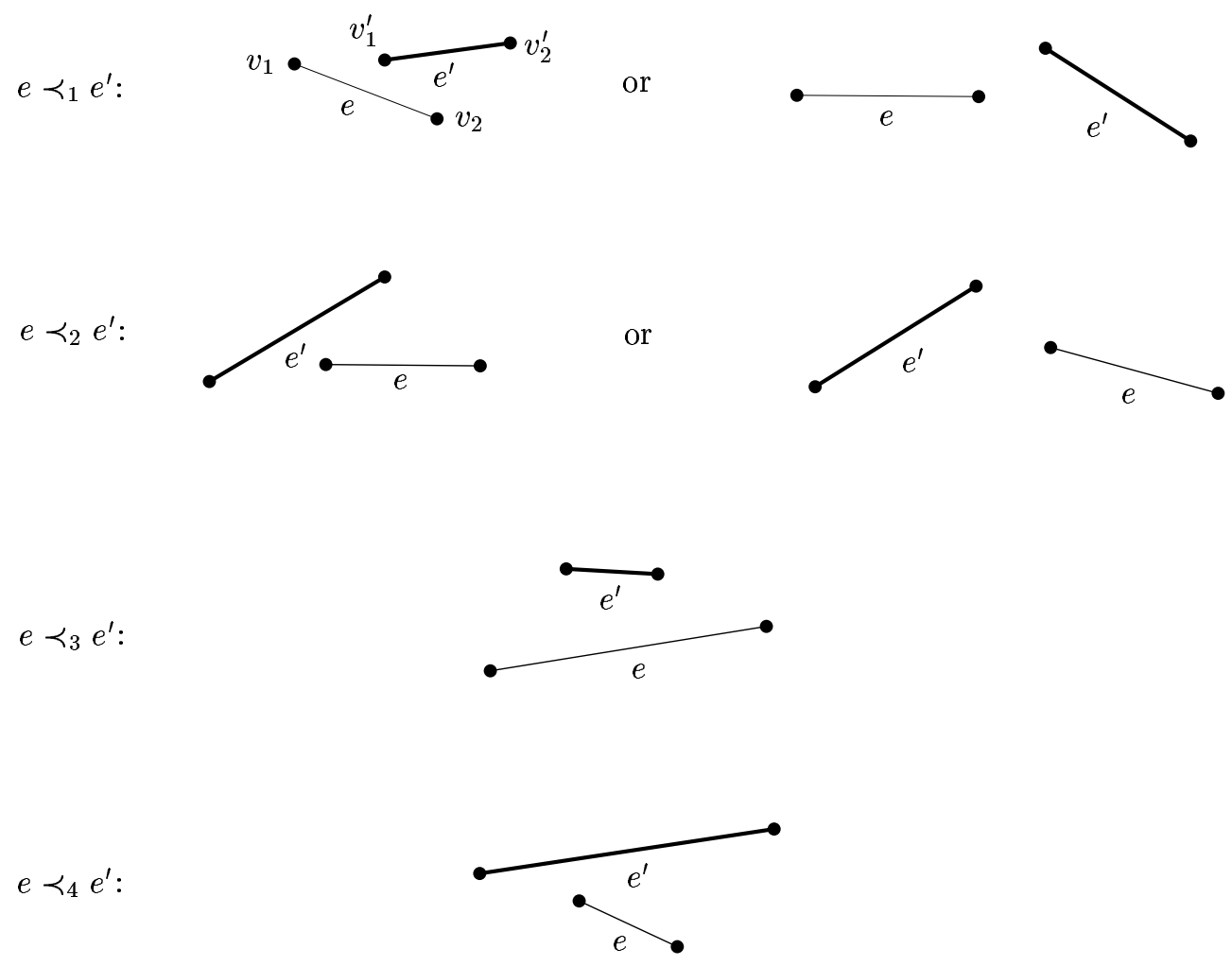

Figure 2: The relations $\prec_{i}$.

- $e \prec_{4} e^{\prime}$ if $x\left(v_{1}\right)>x\left(v_{1}^{\prime}\right), x\left(v_{2}\right)<x\left(v_{2}^{\prime}\right)$ and $e$ lies below $e^{\prime}$.

Obviously, each of the relations $\prec_{i}$ defines a partial order on $E(G)$, and any pair of disjoint edges is comparable by at least one of them. Since $G$ has no $k$ pairwise disjoint edges, there is no chain of length $k$ in the partially ordered set $\left(E(G), \prec_{i}\right) \quad(1 \leq i \leq 4)$. This implies that, for each $i, E(G)$ can be partitioned into at most $k-1$ classes (antichains) such that no two edges belonging to the same class are comparable by $\prec_{i}$. Overlaying these four partitions, we obtain a decomposition of $E(G)$ into at most $(k-1)^{4}$ classes $E_{j}$ $\left(1 \leq j \leq(k-1)^{4}\right)$ such that no two elements of $E_{j}$ are comparable by any of the relations $\prec_{i}$. Thus, none of the graphs $G_{j}:=\left(V(G), E_{j}\right)$ has two disjoint edges. In view of Theorem 5.2, $\left|E\left(G_{j}\right)\right|=\left|E_{j}\right| \leq n \quad\left(1 \leq j \leq(k-1)^{4}\right)$, and the result follows.

Recently, Tóth improved the above result by showing that the dependence of ex $\left(\mathcal{D}_{k}, n\right)$ on $k$ is at most quadratic. Indeed, he proved a somewhat stronger result.

The length of a path is the number of its edges. Let $\mathcal{P}_{k}$ denote the class of all non-crossing paths of length $k$. Clearly, every non-crossing path of length $2 k-1$ has $k$ pairwise disjoint edges. 
Theorem 5.4 [75]. Let ex $\left(\mathcal{P}_{k}, n\right)$ denote the maximum number of edges that a geometric graph of $n$ vertices can have without containing a non-crossing path of length $k$. Then for every $k$ and $n$, we have

$$
\operatorname{ex}\left(\mathcal{P}_{k}, n\right) \leq C k^{2} n
$$

for a suitable constant $C$.

It is possible, however, that the true order of magnitude of ex $\left(\mathcal{P}_{k}, n\right)$ is $O(k n)$. According to a result of Perles, this holds for convex geometric graphs.

Theorem 5.5 [44]. Let $\operatorname{ex}_{c}\left(\mathcal{P}_{k}, n\right)$ denote the maximum number of edges that a convex geometric graph of $n$ vertices can have without containing a non-crossing path of length $k$. Then for every $n \geq k+1$, we have

$$
\operatorname{ex}_{c}\left(\mathcal{P}_{k}, n\right)=\left\lfloor\frac{(k-1) n}{2}\right\rfloor
$$

A graph that can be obtained by attaching some edges to the vertices of a path is called a caterpillar. In other words, a caterpillar is a tree which contains no three paths of length 2 meeting at a common endpoint. We say that two geometric graphs are of the same type if their underlying abstract graphs are isomorphic. In its most general form, Perles' (unpublished) result is the following.

Theorem 5.6 Let $\mathcal{C} \mathcal{P}_{k}$ denote the class of all non-crossing caterpillars of a fixed type, consisting of $k$ edges. Then for every $n \geq k+1$, we have

$$
\operatorname{ex}_{c}\left(\mathcal{C P} \mathcal{P}_{k}, n\right)=\left\lfloor\frac{(k-1) n}{2}\right\rfloor
$$

We mention a related result for (not necessarily convex) geometric graphs, which can easily be deduced from Theorem 2.2 and from Turán's theorem quoted at the beginning of this section.

Corollary 5.7 Let $\mathcal{O} \mathcal{P}_{k}$ denote the class of all non-crossing outerplanar graphs of a fixed type, which can be obtained from a cycle of length $k$ by adding some (or no) diagonals. Then for every $n \geq k+1$, we have

$$
\operatorname{ex}\left(\mathcal{O P} \mathcal{P}_{k}, n\right)=\operatorname{ex}_{c}\left(\mathcal{O P} \mathcal{P}_{k}, n\right)=\frac{k-2}{2(k-1)}\left(n^{2}-r^{2}\right)+\left(\begin{array}{l}
r \\
2
\end{array}\right)
$$

where $r$ is the remainder of $n$ upon division by $k-1$.

As we pointed out at the end of the preceding section, whenever ex $(\mathcal{H}, n)=$ $O(n)$ holds for a class of forbidden geometric subgraphs $\mathcal{H}$, we can use the idea of the proof of Theorem 3.4 to show that, if $|E(G)|=e \gg \operatorname{ex}(\mathcal{H}, n)$, then $G$ has many subgraphs belonging to $\mathcal{H}$. In particular, Theorem 5.3 can be used to establish the following. 
Theorem 5.8 [55]. For any $k \geq 2$ there exists a constant $c(k)>0$ such that every geometric graph with $n$ vertices and $e \geq k^{4} n$ edges has at least $c(k) e^{2 k-1} / n^{2 k-2} \quad k$-tuples of pairwise disjoint edges.

Theorem 5.4 implies that the last statement also holds with the weaker assumption $e \geq C k^{2} n$.

\section{Ramsey-type results}

In classical Ramsey-theory, one wants to find large monochromatic subgraphs in a complete graph whose edges are coloured with several colours [12],[29]. Most questions of this type can be generalized to complete geometric graphs, where the monochromatic subgraphs are required to satisfy certain geometric conditions.

The next two statements were conjectured by Bialostocki and Dierker and proved by Károlyi, Pach and Tóth.

Theorem 6.1 [42],[43]. If the edges of a finite complete geometric graph are coloured by two colours, there exists a non-crossing spanning tree, all of whose edges are of the same colour.

Proof Let $V=\left\{v_{1}, v_{2}, \ldots, v_{n}\right\}$ denote the vertex set of a complete geometric graph $K_{n}$ whose edges are coloured with red and blue. Suppose without loss of generality that no two vertices have the same $x$-coordinate and that the vertices are listed in increasing order of their $x$-coordinates. The assertion is trivial for $n \leq 2$. Thus, we can assume that $n \geq 3$ and the theorem has already been proved for all complete geometric graphs having fewer than $n$ vertices. We can also assume that all edges along the boundary of the convex hull of $V$ are of the same colour (say, red). Indeed, if two consecutive edges of the convex hull have different colours, then remove their common endpoint from $K_{n}$. By the induction hypothesis, the remaining graph has a monochromatic non-crossing spanning tree. Depending on its colour, this spanning tree can be completed to a monochromatic non-crossing spanning tree of $K_{n}$, by putting back one of the two previously deleted edges of the convex hull of $V$.

For every $i, 1<i<n$, let $K_{i}^{l}$ and $K_{i}^{r}$ denote the subgraphs of $K_{n}$ induced by the points $\left\{v_{1}, \ldots, v_{i}\right\}$ and $\left\{v_{i}, \ldots, v_{n}\right\}$, respectively. By the induction hypothesis, both $K_{i}^{l}$ and $K_{i}^{r}$ have a monochromatic non-crossing spanning tree, $T_{i}^{l}$ resp. $T_{i}^{r}$. We can assume that these two trees have different colours, otherwise their union will meet the requirements of the theorem. We can also assume that $T_{2}^{l}$ is red and $T_{2}^{r}$ is blue. Otherwise, $T_{2}^{r}$ would be red, and it could be completed to a non-crossing red spanning tree of $K_{n}$ by the addition of any edge of the convex hull of $V$ incident to $v_{1}$. Similarly, we can suppose that $T_{n-1}^{l}$ is blue and $T_{n-1}^{r}$ is red. Hence, there exists an $i, 1<i<n-1$, such that

- $T_{i}^{l}$ is red and $T_{i}^{r}$ is blue, 
- $T_{i+1}^{l}$ is blue and $T_{i+1}^{r}$ is red.

Connecting $T_{i}^{l}$ and $T_{i+1}^{r}$ by any edge of the convex hull of $V$ which intersects a vertical line separating $v_{i}$ and $v_{i+1}$, we obtain a non-crossing red spanning tree of $K_{n}$, as required.

Theorem 6.2 [42],[43]. If the edges of a complete geometric graph with $3 n-1$ vertices are coloured by two colours, there exist $n$ pairwise disjoint edges of the same colour. This result is best possible.

The analogues of Theorems 6.1 and 6.2 for abstract graphs, i. e., when the geometric constraints are ignored, were found by Erdős-Rado (see [22]) and Gerencsér and Gyárfás [27], respectively. In fact, Gerencsér and Gyárfás proved the stronger result that for any 2-colouring of the edges of a complete graph with $3 n-1$ vertices, there exists a monochromatic path of length $2 n-1$. This statement, as well as Theorem 6.2 , is best possible, as is shown by the following example. Take the disjoint union of a complete graph of $n-1$ vertices and a complete graph of $2 n-1$ vertices, all of whose edges are red and blue, respectively, and colour all edges between the two parts red.

Theorem 6.2 also has an "asymmetric" version.

Theorem 6.3 [42],[43]. Let $k$ and $l$ be positive integers, $n=k+l+\max \{k, l\}-$ 1. If the edges of a complete geometric graph with $n$ vertices are coloured by red and blue, one can find either $k$ disjoint red edges or $l$ disjoint blue edges. This result cannot be improved.

To formulate some further results of this type, it is convenient to introduce some notation.

Let $\mathcal{G}_{1}$ and $\mathcal{G}_{2}$ be not necessarily different classes of geometric graphs. Let $R\left(\mathcal{G}_{1}, \mathcal{G}_{2}\right)$ denote the smallest positive number $R$ with the property that any complete geometric graph of $R$ vertices, whose edges are coloured with red and blue, contains a geometric subgraph which is either totally red and belongs to $\mathcal{G}_{1}$ or totally blue and belongs to $\mathcal{G}_{2}$. If $\mathcal{G}_{1}=\mathcal{G}_{2}=\mathcal{G}$, we write $R(\mathcal{G})$ instead of $R\left(\mathcal{G}_{1}, \mathcal{G}_{2}\right)$. If we restrict our attention to convex geometric graphs, then the corresponding function is denoted by $R_{c}(\mathcal{G})$. Clearly, $R_{c}(\mathcal{G}) \leq R(\mathcal{G})$.

There is an obvious relationship between $R(\mathcal{G})$ and the function $\operatorname{ex}(\mathcal{G}, n)$ discussed in the preceding sections:

$$
\operatorname{ex}(\mathcal{G}, n)<\frac{1}{2}\left(\begin{array}{l}
n \\
2
\end{array}\right) \Longrightarrow R(\mathcal{G}) \leq n .
$$

Indeed, if we colour the edges of a complete geometric graph with two colours, one of the colour classes will contain at least $\frac{1}{2}\left(\begin{array}{l}n \\ 2\end{array}\right)$ elements. If $\operatorname{ex}(\mathcal{G}, n)<\frac{1}{2}\left(\begin{array}{l}n \\ 2\end{array}\right)$, then there is a geometric graph belonging to $\mathcal{G}$, all of whose edges are in this colour class. Similarly, we have

$$
\operatorname{ex}_{c}(\mathcal{G}, n)<\frac{1}{2}\left(\begin{array}{l}
n \\
2
\end{array}\right) \Longrightarrow R_{c}(\mathcal{G}) \leq n
$$


In the special case when $\mathcal{G}$ is the class $\mathcal{T}_{n}$ of all non-crossing geometric trees of $n$ vertices and the class $\mathcal{D}_{n}$ of all geometric graphs consisting of $n$ disjoint edges, respectively, the values of $R(\mathcal{G})$ are given in Theorems 6.1 and 6.2. Theorem 6.3 provides the exact value of $R\left(\mathcal{G}_{1}, \mathcal{G}_{2}\right)$, when $\mathcal{G}_{1}=\mathcal{D}_{k}$ and $\mathcal{G}_{2}=\mathcal{D}_{l}$.

$$
\begin{gathered}
R\left(\mathcal{T}_{n}\right)=R_{c}\left(\mathcal{T}_{n}\right)=n, \quad R\left(\mathcal{D}_{n}\right)=R_{c}\left(\mathcal{D}_{n}\right)=3 n-1, \\
R\left(\mathcal{D}_{k}, \mathcal{D}_{l}\right)=R_{c}\left(\mathcal{D}_{k}, \mathcal{D}_{l}\right)=k+l+\max \{k, l\}-1
\end{gathered}
$$

Next we show

Theorem 6.4 [44]. Let $\mathcal{C C}_{n}$ and $\mathcal{C C}_{n}^{\prime}$ denote, respectively, the class of all noncrossing cycles of length $n$ and the class of all non-crossing cycles of length $n$ triangulated from a vertex. Then we have

$$
(n-1)^{2}<R_{c}\left(\mathcal{C C}_{n}\right) \leq R\left(\mathcal{C C}_{n}^{\prime}\right) \leq 2(n-1)(n-2)+2
$$

Proof First we prove that $R_{c}\left(\mathcal{C C}_{n}\right)>(n-1)^{2}$. Take $(n-1)^{2}$ points on a circle and partition them into $n-1$ groups, each containing $n-1$ consecutive points. Color red all edges between points in different groups, and colour blue all edges between points belonging to the same group.

Any non-crossing red cycle contains at most one point from each group; hence it cannot have more than $n-1$ points. On the other hand, all vertices of a blue cycle are from the same group, so there is no blue cycle with more than $n-1$ points.

Next we show that $R\left(\mathcal{C C}_{n}^{\prime}\right) \leq 2(n-1)(n-2)+2$. Let $V$ denote the vertex set of a complete geometric graph $K$ of $2(n-1)(n-2)+2$ vertices, whose edges are coloured with red and blue. Let $v$ be a vertex of the convex hull of $V$. Since there are $2(n-1)(n-2)+1$ edges incident to $v$, at least $(n-1)(n-2)+1$ of them are of the same colour, say, red. Let $v_{1}, v_{2}, \ldots, v_{(n-1)(n-2)+1}$ be the vertices of $K$, listed in clockwise order of visibility from $v$, such that each edge $v v_{i}$ is red. A path $v_{i_{1}} v_{i_{2}} \ldots v_{i_{j}}$ is said to be monotone if $i_{1}<i_{2}<\ldots<i_{j}$.

Define a partial ordering of the vertices $v_{1}, v_{2}, \ldots, v_{(n-1)(n-2)+1}$, as follows. Let $v_{i}<v_{j}$ if $i<j$ and there is a monotone red path connecting $v_{i}$ to $v_{j}$. As in the proof of Theorem 5.3, there are either $n-1$ elements that form a chain (totally ordered subset), or $n$ elements that form an antichain (i.e. they are pairwise incomparable). In the former case, there is a monotone red path $u_{1}, u_{2}, \ldots, u_{n-1}$, and we can complete it to a non-crossing red cycle $v, u_{1}, u_{2}, \ldots, u_{n-1}$ of size $n$, together with the corresponding diagonals from $v$. In the latter case, there is a complete blue subgraph of $n$ vertices, $u_{1}, u_{2}, \ldots, u_{n}$, because any two incomparable elements are connected by a blue edge. By Theorem 2.2, this complete blue subgraph contains a non-crossing cycle of length $n$, triangulated from a vertex. 
Corollary 6.5 [44]. For any 2-colouring of the edges of a complete geometric graph with $n$ vertices, there exist monochromatic non-crossing cycles of length $3,4, \ldots,\lfloor\sqrt{n / 2}\rfloor$, having the same colour. The order of magnitude of this bound cannot be improved.

Let $\mathcal{P}_{n}$ denote the class of all non-crossing paths of length $n$. It is an immediate consequence of the above results that

$$
R\left(\mathcal{P}_{n}\right) \leq R\left(\mathcal{C C}_{n+1}\right) \leq 2 n(n-1)+2
$$

However, we have a better upper bound on $R\left(\mathcal{P}_{n}\right)$.

Theorem 6.6 [44]. For any 2-colouring of the edges of a complete geometric graph with $n$ vertices, there exists monochromatic non-crossing path whose length is at least a constant times $n^{2 / 3}$. Equivalently,

$$
R\left(\mathcal{P}_{n}\right)=O\left(n^{3 / 2}\right)
$$

It is very likely that $R\left(\mathcal{P}_{n}\right)$ is linear in $n$. For convex geometric graphs we can verify this conjecture.

Theorem 6.7 [44]. For any 2-colouring of the edges of a convex complete geometric graph with $n \geq 3$ vertices, there exists monochromatic non-crossing path of length $\left\lfloor\frac{n+1}{2}\right\rfloor$. This bound cannot be improved. Consequently, for every $n$, we have

$$
R_{c}\left(\mathcal{P}_{n}\right)=2 n-1
$$

Proof We prove only the easy part of (3): if $n$ is even then $R_{c}\left(\mathcal{P}_{n}\right) \leq 2 n-1$. In view of (2) and Theorem 5.6, it is enough to verify that

$$
\left\lfloor\frac{(n-1)(2 n-1)}{2}\right\rfloor<\frac{1}{2}\left(\begin{array}{c}
2 n-1 \\
2
\end{array}\right)
$$

which is trivially true.

Some other Ramsey numbers for convex geometric graphs were studied by H. Harborth and H. Lefmann [34].

For any positive integer $n$, let $n \mathcal{G}$ denote the class of all geometric graphs that can be obtained by taking the union of $n$ pairwise disjoint members of $\mathcal{G}$, any two of which can be separated by a straight line.

Theorem 6.8 [44]. Let $\mathcal{G}$ be any class of geometric graphs, each of which has at least two vertices.

1. If $n$ is a power of 2 then

$$
R(n \mathcal{G}) \leq(R(\mathcal{G})+1) n-1
$$


2. For any $n>0$,

$$
R(n \mathcal{G}) \leq\left\lceil\frac{3(R(\mathcal{G})+1)}{2}\right\rceil n-\left\lceil\frac{R(\mathcal{G})+1}{2}\right\rceil .
$$

3. For any $n>0$,

$$
R_{c}(n \mathcal{G}) \leq\left(R_{c}(\mathcal{G})+1\right) n-1 .
$$

In particular, if $\mathcal{G}=\mathcal{D}_{1}$ is the class of all geometric graphs consisting of a single edge, then $R\left(\mathcal{D}_{1}\right)=2$, and part 1 of Theorem 6.8 implies that

$$
R\left(n \mathcal{D}_{1}\right)=R\left(\mathcal{D}_{n}\right) \leq 3 n-1,
$$

provided that $n$ is a power of 2 . Theorem 6.2 shows that this inequality holds for every $n$, and it is best possible.

Suppose next that $\mathcal{G}=\Delta\left(=\mathcal{C C}_{3}\right)$ is the class of all triangles. Then we have $R(\Delta)=6$, so, by part 1 of Theorem 6.8 , now we obtain

$$
R(n \Delta) \leq 7 n-1,
$$

provided that $n$ is a power of 2 . The following theorem shows that this result cannot be improved.

Theorem 6.9 [44] Let $\Delta$ denote the class of triangles and let $n$ be a positive integer. Then

$$
R(n \Delta) \geq(R(\Delta)+1) n-1=7 n-1
$$

\section{Applications}

This section outlines a few fairly direct applications of the results presented before. L. Székely [72] discovered that the the following celebrated result of Szemerédi and Trotter readily follows from Theorem 3.4.

Corollary 7.1 [73], [74]. The maximum number of incidences between $m$ distinct lines and $n$ distinct points in the plane is at most

$$
O\left(m^{2 / 3} n^{2 / 3}+m+n\right) .
$$

This bound is tight up to a constant factor.

Proof Assume without loss of generality that every line is incident to at least one point. Let $I$ denote the number of incidences between our points and lines. Define a geometric graph $G$ whose vertex set is the given set of $n$ points, and whose two vertices are connected by a segment if they are consecutive vertices along one of the lines. Clearly, $|E(G)|=I-m$.

On the other hand, by Theorem 3.4, either $|E(G)|<4 n$ or

$$
\mathrm{CR}(G) \geq \frac{1}{33.75} \frac{(I-m)^{3}}{n^{2}}
$$

The result follows by comparing this bound with the trivial relation $\mathrm{CR}(G) \leq$ $\left(\begin{array}{c}m \\ 2\end{array}\right)$. 
The same idea was explored by T. Dey [16] to achieve a breakthrough in the so-called $k$-set problem mentioned in the abstract, which plays an important role in the analysis of many algorithms in computational geometry.

Given a set $P$ of $n$ points in the plane in general position (no 3 on a line), a $k$-element subset $P^{\prime} \subseteq P$ is said to be a $k$-set, if there is an open half-plane $H$ such that $H \cap P=P^{\prime}$. It was shown by Erdős, Lovász, Simmons and Straus [23], [53] that the number of $k$-sets of an $n$-element set is $O\left(n^{3 / 2}\right)$, and this was slightly improved by Pach, Steiger and Szemerédi [62]. The order of magnitude of the best known lower bound is $n \log n$.

Theorem 7.2 [16]. For every $0<k<n$, the number of $k$-sets of an $n$-element point set in general position in the plane is at most $6.5(k+1)^{1 / 3} n$.

Proof Let $P$ be a fixed set of $n$ points in the plane in general position. Let $H_{k}$ be a geometric graph defined on the vertex set $P$ such that two points $u, v \in P$ are connected by a segment if and only if there are precisely $k-1$ points of $P$ strictly on one side of the line $u v$. It is easy to see that the number of edges of $H_{k}$ is equal to the number of $k$-sets of $P$. Orient every edge of $H_{k}$ from left to right, and assume without loss of generality that at least $\left|E\left(H_{k}\right)\right| / 2$ edges have precisely $k-1$ points on their left-hand side. Let $G_{k}$ denote the graph formed by these edges.

Let the successor of an edge $\overrightarrow{u v} \in E\left(G_{k}\right)$ be the edge $\overrightarrow{v w}$ with minimum slope among all edges $v \vec{w}^{\prime}$ whose slopes exceed that of $\overrightarrow{u v}$. This relation arranges the edges of $G_{k}$ into pairwise disjoint convex chains. It is not hard to verify that there is precisely one chain starting (ending) at each of the $k$ leftmost (rightmost) points of $P$, and no chain starts (ends) anywhere else.

Assign to each crossing $p \notin V(G)$ of two chains, $\gamma_{1}$ and $\gamma_{2}$, the uniquely determined segment $u_{p} v_{p}$ below $p$, whose supporting line is a common tangent to $\gamma_{1}$ and $\gamma_{2}$. (We say that a segment is below $p$ if it intersects the half-line starting at $p$ and pointing to the direction of the negative $y$-axis.) Clearly, $u_{p}, v_{p} \in P$, and no segment is assigned to two different crossings. The number of tangent segments assigned to crossings is at most $k n$, because each vertex occurs at most once as the left endpoint of a tangent segment, whose other endpoint lies on a chain not passing through $p$. Thus, the total number of crossings,

$$
\mathrm{CR}\left(G_{k}\right) \leq n k .
$$

On the other hand, by Theorem 3.4, either $\left|E\left(G_{k}\right)\right|<4 n$ or

$$
\mathrm{CR}\left(G_{k}\right) \geq \frac{1}{33.75} \frac{\left|E\left(G_{k}\right)\right|^{3}}{n^{2}}
$$

Comparing these two bounds, we obtain

$$
\left|E\left(H_{k}\right)\right| \leq 2\left|E\left(G_{k}\right)\right| \leq 6.5(k+1)^{1 / 3} n
$$


The proofs of Theorems 3.4 and 3.6 easily generalize to arbitrary graph drawings, where the arcs representing the edges are not necessarily straightline segments. Using these generalizations, the next two theorems, due to Clarkson, Edelsbrunner, Guibas, Sharir and Welzl [15] and Spencer, Szemerédi and Trotter [70], can be established by minor modifications of the proof of Corollary 7.1.

Corollary 7.3 [15], [18]. The total number of sides of $m$ distinct cells in an arrangement of $n$ lines in the plane is at most $O\left(m^{2 / 3} n^{2 / 3}+m\right)$.

Corollary $7.4[70]$.The number of unit distances determined by $n$ points in the plane is $O\left(n^{4 / 3}\right)$.

A family $\Gamma$ of curves in the plane is said to have $d$ degrees of freedom if there exists an integer $s$ such that

- no two curves in $\Gamma$ have more than $s$ points in common, and

- for any $d$ points, there are at most $s$ curves in $\Gamma$ passing through all of them.

Pach and Sharir applied the above mentioned (straightforward) extension of Theorem 3.4 to obtain the following common generalization of Corollaries 7.1 and 7.4.

Theorem 7.5 [60], [59]. Let $\Gamma$ be a family of curves in the plane with d degrees of freedom. Then the maximum number of incidences between $n$ points in the plane and $m$ elements of $\Gamma$ is

$$
O\left(n^{d /(2 d-1)} m^{(2 d-2) /(2 d-1)}+n+m\right) .
$$

Using the same technique, Székely slightly improved a well-known result of Chung, Szemerédi and Trotter [14].

Theorem 7.6 [72]. Any set of $n$ points in the plane has an element, from which there are at least $\mathrm{cn}^{4 / 5}$ distinct distances to the remaining points. (Here $c>0$ is an absolute constant.)

Next we present some easy consequences of Theorem 3.6 and its extension to arbitrary graph drawings. The proof of Corollary 4.2 was also based on this result.

Corollary 7.7 The number of crossings in an arbitrary drawing of a random graph with $n$ vertices and $e \geq 4 n$ edges is at least $C e^{2}$, where $C>0$ is a suitable constant. This bound is evidently tight, apart from the constant. 
Proof It is sufficient to verify that the bisection width of such a random graph is almost surely at least constant times $e$.

A drawing of a graph is called polygonal if every edge is represented by a non-crossing polygonal path. In Section 2 , we saw that every outerplanar graph of $n$ vertices admits a straight-line drawing without crossing such that its vertex set is mapped into an arbitrarily prespecified $n$-element set (Theorem 2.2). If we also want to specify the location of each vertex, then usually we cannot insist on straight-line edges. Of course, even in this case we can always find a crossing-free polygonal drawing. We want to minimize the total length (i.e. the total number of segments) of the polygonal paths representing the edges.

Corollary $7.8[66]$. For every $n$, there exist an abstract outerplanar graph $G_{n}$ of $n$ vertices and an assignment of locations (distinct points) for the vertices such that in any planar drawing of $G_{n}$ with the property that each vertex is mapped into the point assigned to it and each edge is represented by a polygonal path, there are at least $n / 100$ edges consisting of at least $n / 100$ segments.

Proof We show only the weaker assertion that there exist a $G_{n}$ and an assignment of locations such that the total number of segments forming the edges of $G_{n}$ in any polygonal drawing of $G_{n}$ with the given point locations, is at least constant times $n^{2}$.

Let $G_{n}$ be a matching of size $\lfloor n / 2\rfloor$, and let the set of locations be the vertex set $P$ of a regular $n$-gon. Randomly assign to each vertex of $G_{n}$ a distinct element of $P$. Assume that with this assignment the matching can be realized with pairwise disjoint polygonal paths of total length $L$. Add to this drawing the edges of the convex hull of $P$. In this way, we produce a polygonal drawing of a graph $G_{n}^{\prime}$ obtained from a cycle by adding a random set of $\lfloor n / 2\rfloor$ vertex-disjoint diagonals. As every edge of a polygonal path crosses at most two convex hull edges, the number of crossings in this drawing is at most $2 L$. On the other hand, it is easy to see that, almost surely, the bisection width of $G_{n}^{\prime}$ is at least $C n$, for a suitable positive constant $C$. By the obvious generalization of Theorem 3.6 to polygonal drawings, we have that the number of crossings is at least

$$
\frac{1}{40} C^{2} n^{2}-\frac{1}{16} \sum_{i=1}^{n} 3^{2}>\frac{1}{50} C^{2} n^{2},
$$

provided that $n$ is large enough. Thus, with probability tending to 1 as $n \rightarrow \infty$, we have $L>n C^{2} / 100$.

Pach and Wenger [66] also designed a drawing algorithm for arbitrary planar graphs, with the property that every edge is represented by a polygonal path of length $O(n)$.

The proof of the following result is almost identical to the last argument. 
Corollary 7.9 [58]. There exist two point sets $\left\{p_{1}, \ldots, p_{n}\right\},\left\{q_{1}, \ldots, q_{n}\right\}$ in the plane with the property that any piecewise linear homeomorphism from the plane to the itself, for which $f\left(p_{i}\right)=q_{i}(i=1, \ldots, n)$, consists of at least constant times $n^{2}$ linear pieces.

D. Souvaine and R. Wenger [69] showed that there always exists a homeomorphism meeting the requirements of the last corollary, which consists of $O\left(n^{2}\right)$ linear pieces.

Finally, we mention an old application of Theorem 5.2.

Corollary 7.10 [36]. The maximum number of point pairs realizing the diameter of a $n$-element set in the plane is equal to $n$.

Proof It is enough to notice that connecting two elements of a set $P$ by a segment if their distance is equal to the diameter of $P$, we obtain a geometric graph with no two disjoint edges. The result follows from Theorem 5.2.

In view of this statement, there is always a point whose degree in the graph of diameters is at most 2. Thus, the chromatic number of this graph is at most 3 , and the diameter of each colour class is smaller than 1 . This is a very special case of Borsuk's famous conjecture [11], which turned out to be false in high dimensions [38].

Corollary 7.11 Every finite point set in the plane can be partitioned into three subsets of smaller diameter.

Z. Füredi [26] applied a Turán-type result with a class of forbidden 5-point geometric subgraphs to prove that the maximum number of times that the same distance can occur among $n$ vertices of a convex polygon is $O(n \log n)$. The best known lower bound, $2 n-7$, is due to Edelsbrunner and Hajnal [19]. Erdős and L. Moser conjectured that the true order of magnitude of this function is $\Theta(n)$.

\section{Geometric hypergraphs}

It seems plausible that to extend the incidence results to higher dimensions, to improve the upper bound for the number of times the unit distance can occur among $n$ points in 3-space, or to make progress concerning the higher dimensional analogue of the $k$-set problem, one has to find the right generalizations of the results discussed in the preceding sections to systems of surfaces or surface patches in $d$-space. For simplicity, we will discuss only the case when these surface patches are flat (simplices).

Definition Ad-dimensional geometric $r$-hypergraph $H_{r}^{d}$ is a pair $(V, E)$, where $V$ is a set of points in general position in $\mathbb{R}^{d}$, and $E$ is a set of closed $(r-1)$ dimensional simplices induced by some $r$-tuples of $V$. The sets $V$ and $E$ are called the vertex set and edge set of $H_{r}^{d}$, respectively. 
Clearly, a geometric graph is a 2-dimensional geometric 2-hypergraph.

Given any class $\mathcal{F}$ of forbidden geometric hypergraphs, let $\operatorname{ex}_{r}^{d}(\mathcal{F}, n)$ denote the maximum number of edges that a $d$-dimensional geometric $r$-hypergraph $H_{r}^{d}$ of $n$ vertices can have without containing a geometric subhypergraph which belongs to $\mathcal{F}$. Most bounds in this section are asymptotic: $d$ and $r$ are thought to be fixed, while $n$ tends to infinity.

Akiyama and Alon proved the following theorem.

Theorem 8.1 [4]. Let $V=V_{1} \cup \ldots \cup V_{d}\left(\left|V_{1}\right|=\ldots=\left|V_{d}\right|=n\right)$ be a $d n$-element set in general position in $\mathbb{R}^{d}$, and let $E$ consist of all $(d-1)$ dimensional simplices having exactly one vertex in each $V_{i}$. Then $E$ contains $n$ disjoint simplices.

According to a well-known result of Erdős [21], any abstract $d$-uniform hypergraph with $n$ vertices and at least $n^{d-(1 / k)^{d-1}}$ hyperedges contains a complete $d$-partite subhypergraph with $k$ elements in each of its classes. Combining this with the previous result, we obtain

Theorem 8.2 [4] Let $\mathcal{D}_{k}^{d}$ denote the class of all $d$-dimensional geometric $d$ hypergraphs consisting of $k$ pairwise disjoint edges. Then the maximum number of hyperedges that a $d$-dimensional geometric d-hypergraph of $n$ vertices can have without containing $k$ pairwise disjoint hyperedges, satisfies

$$
\operatorname{ex}\left(\mathcal{D}_{k}^{d}, n\right)<n^{d-(1 / k)^{d-1}} \text {. }
$$

However, it is conjectured that the true order of magnitude of ex $\left(\mathcal{D}_{k}^{d}, n\right)$ is $O\left(n^{d-1}\right)$. Theorems 5.3 and 5.4 show that this is the case when $d=2$.

If we want to exclude crossings rather than disjoint edges, or want to generalize Theorem 3.4 to geometric hypergraphs, we face the following problem. Even if we restrict our attention to systems of triangles induced by 3-dimensional point sets in general position, it is not completely clear how a "crossing" should be defined. If two segments cross, they do not share an endpoint. Should this remain true for triangles? We have to clarify the terminology.

Definition Two simplices are said to have a non-trivial intersection, if their relative interiors have a point in common. If, in addition, the two simplices are vertex disjoint, then they are said to cross.

More generally, $k$ simplices are said to have a non-trivial intersection, if their relative interiors have a point in common. If, in addition, all simplices are vertex disjoint, then they are said to cross.

Consider $k$ simplices. The fact that every pair of them has a non-trivial intersection does not imply that all of them do. To emphasize that this stronger condition is satisfied, we often say that the simplices have a non-trivial intersection in the strong sense, or simply that they strongly intersect. Similarly, 
a set of pairwise crossing simplices is not necessarily crossing. If we want to emphasize that they all cross, we say that they cross in the strong sense, or, in brief, that they strongly cross.

Definition Let $\mathcal{I}_{k}^{r}$ (respectively, $\mathcal{S I}_{k}^{r}$ ) denote the class of all geometric hypergraphs consisting of $k(r-1)$-dimensional simplices, any two of which have a non-trivial intersection (respectively, all of which are strongly intersecting). Similarly, let $\mathcal{C}_{k}^{r}$ (respectively, $\mathcal{S C}_{k}^{r}$ ) denote the class of all geometric hypergraphs consisting of $k$ pairwise crossing (respectively, strongly crossing) $(r-1)$-simplices.

Theorem 8.3 [8],[18]. For any fixed $k>1$, one can select at most $O\left(n^{\lceil d / 2\rceil}\right)$ $d$-dimensional simplices induced by $n$ points in $d$-space with the property that no $k$ of them share a common interior point. This bound cannot be improved. That is,

$$
\operatorname{ex}_{d+1}^{d}\left(\mathcal{I}_{k}^{d+1}, n\right)=\Theta\left(n^{\lceil d / 2\rceil}\right), \quad \operatorname{ex}_{d+1}^{d}\left(\mathcal{S I}_{k}^{d+1}, n\right)=\Theta\left(n^{\lceil d / 2\rceil}\right) .
$$

Proof Clearly, we have

$$
\Omega\left(n^{\lceil d / 2\rceil}\right) \leq \operatorname{ex}_{d+1}^{d}\left(\mathcal{I}_{k}^{d+1}, n\right) \leq \operatorname{ex}_{d+1}^{d}\left(\mathcal{S I}_{k}^{d+1}, n\right)
$$

where the first inequality follows from the fact that there are triangulations of size $\Omega\left(n^{\lceil d / 2\rceil}\right)$ with $n$ vertices in $\mathbb{R}^{d}$. Consider, for example, the vertical projection of the lower part of any cyclic polytope of $n$ vertices in $\mathbb{R}^{d+1}$.

To see that $\operatorname{ex}_{d+1}^{d}\left(\mathcal{S I}_{k}^{d+1}, n\right) \leq O\left(n^{\lceil d / 2\rceil}\right)$, we set up a charging scheme. Let us regard $\mathbb{R}^{d-1}$ as the coordinate hyperplane in $\mathbb{R}^{d}$ spanned by the first $d-1$ axes, and let $X_{d}$ denote the last coordinate axis. Suppose that $X_{d}$ is vertical. Fix a geometric hypergraph $H_{d+1}^{d}=(V, E)$ which has no $k$ edges with a common interior point and whose $n$ vertices are in general position. For any l-dimensional simplex $\Delta$ induced by $V$, where $l \leq\lfloor(d-1) / 2\rfloor$, let $E_{\Delta} \subseteq E$ denote the set of all edges of $H_{d+1}^{d}$ that contain $\Delta$ on their boundaries. It follows from the condition on $H_{d+1}^{d}$ that the infinite vertical cylinder $\Delta+X_{d}$ based on $\Delta$ intersects the interior of at most $2(k-1)$ elements of $E_{\Delta}$. Let us charge $\Delta$ one unit for each of these edges. Since the total number of $l$-simplices with $l \leq\lfloor(d-1) / 2\rfloor$ is at most $\lceil d / 2\rceil n^{\lceil d / 2\rceil}$, it remains to show that every edge $e \in E$ has been charged for. Indeed, by Radon's theorem [67], the vertex set of the orthogonal projection of $e$ into $\mathbb{R}^{d-1}$ can be partitioned into two parts, $S_{1}$ and $S_{2}$, such that their convex hulls cross each other and $\left|S_{1}\right|+\left|S_{2}\right|=d+1$. Suppose without loss of generality that $\left|S_{1}\right| \leq\lfloor(d+1) / 2\rfloor$. Then the convex hull of $S_{1}$ is an $l$-dimensional simplex $\Delta_{1}$ for some $l \leq\lfloor(d-1) / 2\rfloor$, and we had to charge $\Delta_{1}$ for $e$. 
Theorem 8.4 [18]. Let $E$ be any set of d-dimensional simplices induced by an n-element point set $V \subseteq \mathbb{R}^{d}$. If $E$ has no two crossing elements, then $|E|=O\left(n^{d}\right)$, and this bound is asymptotically tight. In notation,

$$
\operatorname{ex}_{d+1}^{d}\left(\mathcal{C}_{2}^{d+1}, n\right)=\Theta\left(n^{d}\right) .
$$

The last two theorems were about $d$-dimensional geometric $d+1$-hypergraphs, i.e., systems of full-dimensional simplices induced by a set of points.

Next we present a few statements on $d$-dimensional geometric $d$-hypergraphs. They can be viewed as natural extensions of the corresponding results for geometric graphs (the case $d=2$ ) discussed in the preceding sections.

Theorem 8.5 [18],[79]. Let $E$ be a family of $(d-1)$-dimensional simplices induced by an $n$-element point set $V \subseteq \mathbb{R}^{d}$ such that $E$ has no $k$ members with pairwise non-trivial intersections $(d, k>1)$. Then, for $k=2$ and 3 , we have $|E|=O\left(n^{d-1}\right)$. Otherwise, $|E|=O\left(n^{d-1} \log n\right)$. In notation,

$$
\begin{gathered}
\operatorname{ex}_{d}^{d}\left(\mathcal{I}_{k}^{d}, n\right)=O\left(n^{d-1}\right) \quad \text { if } k=2,3 ; \\
\operatorname{ex}_{d}^{d}\left(\mathcal{I}_{k}^{d}, n\right)=O\left(n^{d-1} \log n\right) \quad \text { otherwise. }
\end{gathered}
$$

This result is asymptotically tight if $d, k \leq 3$.

Proof For $d=2$, the assertion is true, by Theorem 4.1 and by Corollary 4.4. Assume that $d \geq 3$. For any $(d-3)$-simplex $\Delta$ induced by $V$, let $E_{\Delta}$ denote the family of all members of $E$ that contain $\Delta$ as a face. Pick any point $p_{\Delta}$ in the relative interior of $\Delta$, and let $F_{\Delta}$ denote the 3-dimensional flat orthogonal to $\Delta$ and passing through $p_{\Delta}$.

Every $e \in E_{\Delta}$ meets $F_{\Delta}$ in a polygon, whose two sides incident to $p_{\Delta}$ are the intersections of $F_{\Delta}$ with the two $(d-2)$-faces of $e$ containing $\Delta$. Thus, the total number of sides incident to $p_{\Delta}$ that occur in some $e \cap F_{\Delta}\left(e \in E_{\Delta}\right)$ is at most $n-d+2<n$. Take a small 2-dimensional sphere $S^{2} \subseteq F_{\Delta}$ centered at $p_{\Delta}$. The intersections of $S^{2}$ with the elements of $E_{\Delta}$ form the edge set of a graph with at most $n$ vertices. It follows from the properties of $E$ that this graph has no $k$ pairwise crossing edges, so, by the planar results, its number of edges, $\left|E_{\Delta}\right|$, satisfies

$$
\begin{gathered}
\left|E_{\Delta}\right|=O(n) \quad \text { if } k=2,3 \\
\left|E_{\Delta}\right|=O(n \log n) \quad \text { otherwise. }
\end{gathered}
$$

Summing over all $(d-3)$-simplices $\Delta$ induced by $V$, we obtain $\left(\begin{array}{l}d \\ 2\end{array}\right)|E|=$ $\sum_{\Delta}\left|E_{\Delta}\right|$, and hence the upper bound.

To show that the result is tight for $d=3, k=2$, consider a nested sequence of $n / 2$ pyramids based on the same 2 -dimensional convex $n / 2$-gon. These pyramids have a total of $n^{2} / 4$ triangular faces, no two of which have a nontrivial intersection. 
It is an outstanding open problem to decide whether the order of magnitude of the above bound can be improved, e.g., for $d=4, k=2$. However, we have the following related result.

Theorem 8.6 [18]. Let $E$ be a family of $(d-1)$-dimensional simplices induced by an $n$-element point set $V \subseteq \mathbb{R}^{d}$. If $E$ has no two crossing members, then $|E|=O\left(n^{d-1}\right)$, and this bound cannot be improved. In notation,

$$
\operatorname{ex}_{d}^{d}\left(\mathcal{C}_{2}^{d}, n\right)=\Theta\left(n^{d-1}\right)
$$

This can be generalized as follows.

Theorem 8.7 Let $E$ be a family of $(d-1)$-dimensional simplices induced by an $n$-element point set $V \subseteq \mathbb{R}^{d}$, where $d, k>1$. If $E$ has no $k$ pairwise crossing members, then $|E|=O\left(n^{\bar{d}-(1 / d)^{k-2}}\right)$. In notation,

$$
\operatorname{ex}_{d}^{d}\left(\mathcal{C}_{k}^{d}, n\right)=O\left(n^{d-(1 / d)^{k-2}}\right)
$$

The proof of this result is based on a natural extension of Theorem 4.8, which provides us with a recipe for giving a lower bound on the number of crossing $k$-tuples of edges, if we know how many edges are necessary to guarantee the existence of one such $k$-tuple.

Theorem 8.8 [18]. Assume that, for suitable constants $c_{1}$ and $0 \leq \delta \leq 1$, we have $\operatorname{ex}_{r}^{d}\left(\mathcal{S C}_{k}^{r}, n\right)<c_{1}\left(\begin{array}{l}n \\ r\end{array}\right) / n^{\delta}$ and $e \geq\left(c_{1}+1\right)\left(\begin{array}{l}n \\ r\end{array}\right) / n^{\delta}$.

Then there exists $c_{2}>0$ such that the minimum number of strongly crossing $k$-tuples of edges in a $d$-dimensional $r$-hypergraph with $n$ vertices and $e$ edges is at least

$$
c_{2}\left(\begin{array}{c}
n \\
k r
\end{array}\right) e^{\gamma} /\left(\begin{array}{l}
n \\
r
\end{array}\right)^{\gamma}
$$

where $\gamma=1+\frac{(k-1) r}{\delta}$.

As Theorem 3.4 can be applied to the planar $k$-set problem (compare Theorem 7.2), the last result, which is a far-reaching generalization of Theorem 3.4, is relevant to its higher dimensional analogues.

Given a set $P$ of $n$ points in 3-space, in general position, a $k$-element subset $P^{\prime} \subseteq P$ is called a $k$-set, if there is a half-space $H$ such that $H \cap P=P^{\prime}$. Bárány, Füredi and Lovász [9] gave the first non-trivial (subcubic) upper bound for the number of $\lfloor n / 2\rfloor$-sets of an $n$-element set in 3 -space. The best known bound is given by the following theorem.

Theorem 8.9 [17], [6]. The number of $\lfloor n / 2\rfloor$-sets of an n-element set in general position in 3 -space is $O\left(n^{8 / 3}\right)$. 
Proof Let $P$ be a set of $n$ points in general position in 3-space. Similarly to the 2-dimensional case, it is sufficient to bound the number of edges in the 3dimensional geometric 3-hypergraph $H=(V, E)$, where $V=P$ and $E$ consists of all triangles whose supporting planes pass through 3 elements of $P$, and which have precisely $\lfloor n / 2\rfloor$ points on one of their sides. Let $e:=|E|$.

In view of Theorem 8.6, we can apply Theorem 8.8 with $d=r=3, k=2$, and $\delta=1$ to obtain that the number of (strongly) crossing pairs of edges of $H$ is at least $\Omega\left(e^{4} / n^{6}\right)$, provided that $e>c n^{2}$ for a suitable constant $c>0$. Notice that if two triangles cross each other, then one of them must have an edge that crosses the other triangle. Thus, there is an edge crossing at least $\Omega\left(e^{3} / n^{6}\right)$ triangles. On the other hand, by an old (and easy) observation of Lovász, every straight line crosses at most $n^{2}$ edges of $H$. We have

$$
n^{2}=\Omega\left(e^{3} / n^{6}\right)
$$

and the result follows.

We close this section with a generalization of Theorem 6.2.

Theorem 8.10 [18]. Let us colour with two colours all $(d-1)$-dimensional simplices induced by $(d+1) n-1$ points in general position in $\mathbb{R}^{d}$. Then one can always find $n$ disjoint simplices of the same colour. This result cannot be improved.

\section{Acknowledgements}

I thank Professor Micha Perles, who gave a course on geometric graph theory at Rutgers University in 1989, during the Special Year in Discrete and Computational Geometry, the first "special year" of DIMACS (Center for Discrete Mathematics and Theoretical Computer Science). Many of the results surveyed here grew out of discussions following his exciting lectures ten years ago.

This research was supported by National Science Foundation grant CCR9732101, by PSC-City University of New York Research Award 667339, and by a grant from the Hungarian National Fund for Scientific Research (T-020914).

\section{References}

[1] M. Abellanas, J. García, G. Hernández, M. Noy \& P. Ramos, Bipartite embeddings of trees in the plane, in Graph Drawing '96 (ed. S. North), Lecture Notes in Computer Science, 1190, Springer-Verlag, Berlin (1997), pp. $1-10$.

[2] P. K. Agarwal, B. Aronov, J. Pach, R. Pollack \& M. Sharir, Quasi-planar graphs have a linear number of edges, Combinatorica, 17 (1997), 1-9. 
[3] M. Ajtai, V. Chvátal, M. Newborn \& E. Szemerédi, Crossing-free subgraphs, Annals of Discrete Mathematics, 12 (1982), 9-12.

[4] J. Akiyama \& N. Alon, Disjoint simplices and geometric hypergraphs, in Combinatorial Mathematics (eds. G. S. Bloom et al.), Annals of New York Academy of Sciences, 555, (1989), pp. 1-3.

[5] N. Alon \& P. Erdős, Disjoint edges in geometric graphs, Discrete and Computational Geometry, 4 (1989), 287-290.

[6] B. Aronov, B. Chazelle, H. Edelsbrunner, L. Guibas, M. Sharir \& R. Wenger, Points and triangles in the plane and halving planes in space, Discrete and Computational Geometry, 6 (1991), 435-442.

[7] S. Avital \& H. Hanani, Graphs (in Hebrew), Gilyonot Lematematika, 3 (1966), 2-8.

[8] I. Bárány \& Z. Füredi, Empty simplices in Euclidean space, Canadian Mathematical Bulletin, 30 (1987), 436-445.

[9] I. Bárány, Z. Füredi \& L. Lovász, On the number of halving planes, Combinatorica, 10 (1990), 175-183.

[10] B. Bollobás, Extremal Graph Theory, Academic Press, New York (1978).

[11] K. Borsuk, Drei Sätze über die $n$-dimensionale euklidische Sphäre, Fundamenta Mathematicae, 20 (1933), 177-190.

[12] S. Burr, Generalized Ramsey-theory for graphs - a survey, in Graphs and Combinatorics (eds. R. Bari \& F. Harary), Lecture Notes in Mathematics, 406, Springer-Verlag, Berlin (1974), pp. 52-75.

[13] V. Capoyleas \& J. Pach, A Turán-type theorem on chords of a convex polygon, Journal of Combinatorial Theory, Series B, 56 (1992), 9-15.

[14] F. Chung, E. Szemerédi \& W. T. Trotter, The number of distinct distances determined by a set of points in the Euclidean plane, Discrete and Computational Geometry, 7 (1992), 1-11.

[15] K. Clarkson, H. Edelsbrunner, L. Guibas, M. Sharir \& E. Welzl, Combinatorial complexity bounds for arrangements of curves and surfaces, Discrete and Computational Geometry, 5 (1990), 99-160.

[16] T. K. Dey, Improved bounds for planar $k$ sets and related problems, Discrete and Computational Geometry, 19 (1998), 373-382.

[17] T. K. Dey \& H. Edelsbrunner, Counting triangle crossings and halving planes, Discrete and Computational Geometry, 12 (1994), 281-289. 
[18] T. K. Dey \& J. Pach, Extremal problems for geometric hypergraphs, Discrete and Computational Geometry, 19 (1998), 473-484.

[19] H. Edelsbrunner \& P. Hajnal, A lower bound on the number of unit distances between the points of a convex polygon, Journal of Combinatorial Theory, Series A, 56 (1991), 312-316.

[20] R. B. Eggleton \& R. K. Guy, The crossing number of the n-cube, Notices of the American Mathematical Society, 17 (1970), 757.

[21] P. Erdős, On extremal problems on graphs and generalized graphs, Israel Journal of Mathematics, 2 (1964), 183-190.

[22] P. Erdős, A. Gyárfás \& L. Pyber, Vertex coverings by monochromatic cycles and trees, Journal of Combinatorial Theory, Series B, 51 (1991), 90-95.

[23] P. Erdős, L. Lovász, A. Simmons \& E. G. Straus, Dissection graphs of planar point sets, in A Survey of Combinatorial Theory (eds. J. N. Srivastava et al.), North-Holland, Amsterdam (1973), pp. 139-149.

[24] I. Fáry, On straight line representation of planar graphs, Acta Scientiarum Mathematicarum (Szeged), 11 (1948), 229-233.

[25] H. deFraysseix, J. Pach \& R. Pollack, How to draw a planar graph on a grid, Combinatorica, 10 (1990), 41-51.

[26] Z. Füredi, The maximum number of unit distances in a convex $n$-gon, Journal of Combinatorial Theory, Series A, 55 (1990), 316-320.

[27] G. Gerencsér \& A. Gyárfás, On Ramsey-type problems, Annales Universitatis Scientarium Budapestinensis Roland Eötvös, Sectio Mathematica, X (1967), 167-170.

[28] W. Goddard, M. Katchalski \& D. J. Kleitman, Forcing disjoint segments in the plane, European Journal of Combinatorics, 17 (1996), 391-395.

[29] R. L. Graham, B. L. Rothschild \& J. H. Spencer, Ramsey Theory, 2nd edition, Wiley, New York (1990).

[30] P. Gritzmann, B. Mohar, J. Pach \& R. Pollack, Embedding a planar triangulation with vertices at specified points (Solution to Problem E3341), American Mathematical Monthly, 98 (1991), 165-166.

[31] A. Gyárfás, On the chromatic number of multiple interval graphs and overlap graphs, Discrete Mathematics, 55 (1985), 161-166.

[32] A. Gyárfás, Corrigendum, Discrete Mathematics, 62 (1986), 333. 
[33] A. Gyárfás \& J. Lehel, Covering and coloring problems for relatives of intervals, Discrete Mathematics, 55 (1985), 167-180.

[34] H. Harborth \& H. Lefmann, Coloring arcs of convex sets, preprint, Technische Universität Braunschweig, 1998.

[35] J. Hershberger \& S. Suri, Applications of a semi-dynamic convex hull algorithm, BIT, 32 (1992), 249-267.

[36] H. Hopf \& E. Pannwitz, Aufgabe No. 167, Jahresbericht der deutschen Mathematiker-Vereinigung, 43 (1934), 114.

[37] Y. Ikebe, M. Perles, A. Tamura \& S. Tokunaga, The rooted tree embedding problem into points in the plane, Discrete and Computational Geometry, 11 (1994), 51-63.

[38] J. Kahn \& G. Kalai, A counterexample to Borsuk's conjecture, Bulletin of the American Mathematical Society, 29 (1993), 60-62.

[39] A. Kaneko \& M. Kano, Straight-line embeddings of two rooted trees in the plane, preprint, Kogakuin University, Tokyo and Ibaraki University, Hitachi, 1998.

[40] A. Kaneko \& M. Kano, Straight-line embeddings of rooted star forests in the plane, preprint, Kogakuin University, Tokyo and Ibaraki University, Hitachi, 1998.

[41] A. Kaneko \& M. Kano, A balanced partition of points in the plane and tree embedding problems, preprint, Kogakuin University, Tokyo and Ibaraki University, Hitachi, 1998.

[42] G. Károlyi, J. Pach \& G. Tóth, Ramsey-type results for geometric graphs, I, Discrete and Computational Geometry, 18 (1997), 247-255.

[43] G. Károlyi, J. Pach, G. Tardos \& G. Tóth, An algorithm for finding many disjoint monochromatic edges in a complete 2-colored geometric graph, in Intuitive Geometry (eds. I. Bárány \& K. Böröczky), Bolyai Society Mathematical Studies, 6, Bolyai Society, Budapest (1997), pp. 367-372.

[44] G. Károlyi, J. Pach, G. Tóth \& P. Valtr, Ramsey-type results for geometric graphs, II, Discrete and Computational Geometry, 20 (1998), 375-388.

[45] M. Klazar \& P. Valtr, Generalized Davenport-Schinzel sequences, Combinatorica, 14 (1994), 463-476.

[46] A. Kostochka, On upper bounds on the chromatic numbers of graphs (in Russian), Transactions of the Institute of Mathematics (Siberian Branch of the Academy of Sciences of the USSR), 10 (1988), 204-226. 
[47] A. Kostochka \& J. Kratochvíl, Covering and coloring polygon-circle graphs, Discrete Mathematics, 163 (1997), 299-305.

[48] Y. Kupitz, Extremal Problems in Combinatorial Geometry, Aarhus University Lecture Notes Series, 53, Aarhus University, Denmark (1979).

[49] Y. Kupitz, On pairs of disjoint segments in convex position in the plane, Annals of Discrete Mathematics, 20 (1984), 203-208.

[50] D. Larman, J. Matoušek, J. Pach \& J. Töröcsik, A Ramsey-type result for planar convex sets, Bulletin of the London Mathematical Society, 26 (1994), 132-136.

[51] T. Leighton, Complexity Issues in VLSI, Foundations of Computing Series, MIT Press, Cambridge, MA (1983).

[52] R. Lipton \& R. Tarjan, A separator theorem for planar graphs, SIAM Journal of Applied Mathematics, 36 (1979), 177-189.

[53] L. Lovász, On the number of halving lines, Annales Universitatis Scientarium Budapestinensis Roland Eötvös, Sectio Mathematica, XIV (1971), 107-108.

[54] S. McGuinness, Colouring arcwise connected sets in the plane, I, preprint, University of Umeå, 1997.

[55] J. Pach, Notes on geometric graph theory, in Discrete and Computational Geometry: Papers from the DIMACS Special Year (eds. J. E. Goodman, R. Pollack \& W. Steiger), DIMACS Series in Discrete Mathematics and Theoretical Computer Science, 6, American Mathematical Society, Providence, RI (1991), pp. 273-285.

[56] J. Pach, Geometric graphs and hypergraphs, Graph Theory Notes of New York, XXXI (1996), 39-43.

[57] J. Pach \& P. K. Agarwal, Combinatorial Geometry, Wiley, New York (1995).

[58] J. Pach, F. Shahrokhi \& M. Szegedy, Applications of the crossing number, Algorithmica, 16 (1996), 111-117.

[59] J. Pach \& M. Sharir, Repeated angles in the plane and related problems, Journal of Combinatorial Theory, Series A, 59 (1990), 12-22.

[60] J. Pach \& M. Sharir, On the number of incidences between points and curves, Combinatorics, Probability and Computing, 7 (1998), 121-127.

[61] J. Pach, J. Spencer \& G. Tóth, New bounds on crossing numbers, preprint, Mathematical Institute of the Hungarian Academy of Sciences, 1999. 
[62] J. Pach, W. Steiger \& E. Szemerédi, An upper bound on the number of planar k-sets, Discrete and Computational Geometry, 7 (1992), 109-123.

[63] J. Pach \& J. Töröcsik, Layout of rooted trees, in Planar Graphs (ed. W. T. Trotter), DIMACS Series in Discrete Mathematics and Theoretical Computer Science, 9, American Mathematical Society, Providence, RI (1993), pp. 131-137.

[64] J. Pach \& J. Töröcsik, Some geometric applications of Dilworth's theorem, Discrete and Computational Geometry, 12 (1994), 1-7.

[65] J. Pach \& G. Tóth, Graphs drawn with few crossings per edge, Combinatorica, 17 (1997), 427-439.

[66] J. Pach \& R. Wenger, Embedding planar graphs with fixed vertex locations, in Graph Drawing '98 Lecture Notes in Computer Science, SpringerVerlag, Berlin (1999), pp. in press..

[67] J. Radon, Mengen konvexer Körper, die einen gemeinsamen Punkt enthalten, Mathematische Annalen, 63 (1921), 113-115.

[68] W. Schnyder, Embedding planar graphs on the grid, in First Annual ACM-SIAM Symposium on Discrete Algorithms SIAM, Philadelphia, PA (1990), pp. 138-147.

[69] D. Souvaine \& R. Wenger, Constructing piecewise linear homeomorphisms, Technical Report 94-52, DIMACS, New Brunswick, NJ, 1994.

[70] J. Spencer, E. Szemerédi \& W. T. Trotter, Unit distances in the Euclidean plane, in Graph Theory and Combinatorics (ed. B. Bollobás), Academic Press, New York (1984), pp. 293-303.

[71] E. Steinitz, Polyeder- und Raumeinteilungen, in Geometrie, Part 3AB12, Enzyklopädie der mathematischen Wissenschaften, 3, (1922), pp. 1-139.

[72] L. A. Székely, Crossing numbers and hard Erdős problems in discrete geometry, Combinatorics, Probability and Computing, 6 (1997), 353-358.

[73] E. Szemerédi \& W. T. Trotter, Extremal problems in discrete geometry, Combinatorica, 3 (1983), 381-392.

[74] E. Szemerédi \& W. T. Trotter, A combinatorial distinction between the Euclidean and projective planes, European Journal of Combinatorics, 4 (1983), 385-394.

[75] G. Tóth, Note on geometric graphs, preprint, Massachusetts Institute of Technology, 1998. 
[76] G. Tóth \& P. Valtr, Geometric graphs with few disjoint edges, in Proceedings of the 14th Annual Symposium on Computational Geometry ACM Press, New York (1998), pp. 184-191.

[77] P. Turán, On the theory of graphs, Colloquium Mathematicum, 3 (1954), 19-30.

[78] W. T. Tutte, How to draw a planar graph, Proceedings of the London Mathematical Society, 13 (1963), 743-768.

[79] P. Valtr, On geometric graphs with no $k$ pairwise parallel edges, Discrete and Computational Geometry, 19 (1998), 461-469.

Mathematical Institute of the Hungarian Academy of Sciences H-1364 Budapest, P.O.B. 127

and

Department of Computer Science City College

City University of New York pach@cims.nyu.edu 